\title{
Probing Efficient Cosmic-Ray Acceleration in Young Supernovae
}

\author{
V. V. Dwarkadas* \\ Department of Astronomy and Astrophysics, University of Chicago, 5640 S Ellis Ave, Chicago, \\ IL 60637 \\ E-mail: vikrameoddjob.uchicago.edu
}

\section{Renaud, A. Marcowith}

Laboratoire Univers et particules de Montpellier, Université Montpellier II/CNRS, place E. Bataillon, cc072, 34095 Montpellier, France

\section{Tatischeff}

Centre de Sciences Nucléaires et de Sciences de la Matière, IN2P3/CNRS and Univ Paris-Sud, 91405 Orsay, France

The formation of a core collapse supernovae ( $\mathrm{SNe}$ ) results in a fast (but non- or mildly-relativistic) shock wave expanding outwards into the surrounding medium. The medium itself is likely modified due to the stellar mass-loss from the massive star progenitor, which may be Wolf-Rayet stars (for Type Ib/c SNe), red supergiant stars (for type IIP and perhaps IIb and IIL SNe), or some other stellar type. The wind mass-loss parameters determine the density structure of the surrounding medium. Combined with the velocity of the SN shock wave, this regulates the shock acceleration process. In this article we discuss the essential parameters that control the particle acceleration and gamma-ray emission in SNe, with particular reference to the Type IIb SN 1993J. The shock wave expanding into the high density medium leads to fast particle acceleration, giving rise to rapidly-growing plasma instabilities driven by the acceleration process itself. The instabilities grow over intraday timescales. This growth, combined with the interplay of non-linear processes, results in the amplification of the magnetic field at the shock front, which can adequately account for the magnetic field strengths deduced from radio monitoring of the source. The maximum particle energy can reach, and perhaps exceed, $1 \mathrm{PeV}$, depending on the dominant instability. The gamma-ray signal is found to be heavily absorbed by pair production process during the first week after the outburst. We derive the time dependent particle spectra and associated hadronic signatures of secondary particles (gamma-ray, leptons and neutrinos) arising from proton proton interactions. We find that the Cherenkov Telescope Array (CTA) should be able to detect objects like SN 1993J above $1 \mathrm{TeV}$. We predict a low neutrino flux above $10 \mathrm{TeV}$, implying a detectability horizon with current or planned neutrino telescopes of $1 \mathrm{Mpc}$.

The 34th International Cosmic Ray Conference

30 July- 6 August, 2015

The Hague, The Netherlands

${ }^{*}$ Speaker. 


\section{Introduction}

Supernova Remnants (SNRs) are widely accepted as the likely source of Galactic cosmic rays (CR) up to energies of $3 \mathrm{PeV}$ (the CR "knee" [1]). Per the Hillas confinement criterion [2] that the Larmor radius of the particle match the source size, only certain Galactic sources should be able to produce such energetic particles. These include extended sources with standard interstellar medium (ISM) magnetic field (MF) values such as massive star clusters and their superbubbles $[3,4,5]$; or compact sources with more intense magnetic fields, such as young SNRs. In this work we explore particle acceleration and gamma-ray radiation in $\mathrm{SNe}$ arising from massive star progenitors, with particular focus on the well-monitored SN 1993J. We consider the acceleration of particles starting from the days following $\mathrm{SN}$ outburst when the radio luminosity is close to its maximum. We review the main results of radio observations and modeling in $\S 2$, examine models of magnetic field amplification (MFA) in SN 1993J in \$2.1, and the implied cosmic ray energies in §2.2. Modeling of different particle distribution species in radio $\mathrm{SNe}$ is outlined in $§ 2.3$. Our calculation of the gamma-ray radiation is shown in $\S 3$, and the neutrino signal in $\$ 3.3$. Some perspectives for other types of $\mathrm{SNe}$, along with conclusions, are discussed in $\S 4$.

\section{A case study: $\mathrm{SN}$ 1993J}

Our model for SN 1993J is based on work by [6](hereafter T09) that discusses particle acceleration in SN 1993J. This Type IIb SN resulted from the explosion of a massive K-supergiant star with an initial mass $\sim 15 M_{\odot}$ [7], having a B-star binary companion [8]. The progenitor star had a mass loss rate $\sim 3.8 \times 10^{-5} M_{\odot} \mathrm{yr}^{-1}$ and a wind velocity $u_{w} \sim 10 \mathrm{~km} \mathrm{~s}^{-1}$ (T09). Assuming a constant mass loss rate, the circumstellar medium (CSM) density scales as $n_{\text {circ }}=\frac{\dot{M}(1+2 X)}{4 \pi r^{2} u_{w} m_{H}(1+4 X)}$ where $X=0.1$ is the Helium fraction and $m_{H}$ the hydrogen atom mass. The effective density downstream of the forward shock, one day after outburst, is $n_{\text {eff }} \simeq 4 \times 10^{9} \mathrm{~cm}^{-3}$ with a shock compression ratio of 4 . The shock radius after 1 day has been estimated as $r \simeq 3.5 \times 10^{14} \mathrm{~cm}$ (T09) from the radio expansion. The shock propagates into a fully ionized medium [9].

The time dependence of the average MF from the synchrotron emitting shell is (T09):

$$
\langle B\rangle \simeq[2.4 \pm 1 \mathrm{G}] \times\left(\frac{\mathrm{t}}{100 \text { days }}\right)^{-1.16 \pm 0.20},
$$

giving a MF of order $500 \mathrm{G}$ after 1 day. This field is consistent with observations [10]. It is an averaged value over the synchrotron shell and does not necessarily represent the MF produced at the forward shock. We identify this field as that in the post shock gas of the forward shock front.

\subsection{Magnetic field amplification (MFA)}

Eq. (2.1) shows that the MF substantially exceeds typical stellar wind fields at these distances. For comparison the equipartition MF in the progenitor wind is [11]:

$$
B_{e q}=\frac{\left(\dot{M} u_{w}\right)^{1 / 2}}{r} \simeq[2.5 \mathrm{mG}] \times \dot{\mathrm{M}}_{-5}^{1 / 2} \times \mathrm{u}_{\mathrm{w}, 10}^{1 / 2} \times \mathrm{r}_{16}^{-1}
$$

about one thousand times less than the value in Eq.2.1. T09 find that the sub-shock compression ratio is close to 4. The compression of the MF given by Eq. (2.2) cannot therefore explain the value 
given by Eq. (2.1) (see also [11]). We conclude and assume that a strong MF amplification process is at work at the forward shock front, driven by the diffusive shock acceleration (DSA) of hadrons.

\subsubsection{Streaming driven instabilities}

Streaming of cosmic rays ahead of the shock front produces magnetic fluctuations. The streaming modes can be in resonance $(\mathrm{R})$ with the energetic particles, i.e. in the high-energy limit they have a wave-number such that $k \sim \mathrm{r}_{\mathrm{L}}^{-1}$; or they can be non-resonant (NR) with a much larger wavenumber [12,13]. The NR modes grow the fastest [14]. For 1993J the NR modes grow and produce magnetic fluctuations over intra-day timescales; we obtain a growth time:

$$
\tau_{N R-s t}=[0.16 \text { day }] \times\left(\frac{\phi / 15}{\left(\xi_{\mathrm{CR}} / 0.05\right) \mathrm{u}_{\mathrm{sh}, 93 \mathrm{~J}}^{3} \sqrt{\mathrm{n}_{93 \mathrm{~J}}}}\right) \times \mathrm{E}_{\mathrm{PeV}} \mathrm{t}_{\text {days }}^{1.17} .
$$

The CR distribution is assumed to scale as $p^{-4}$ over more than 6 orders of magnitude producing $\phi=$ $\ln \left(p_{\max } / p_{\text {inj }}\right)=15$, and $\xi_{C R}=0.05$, so that $5 \%$ of the fluid kinetic energy is imparted to energetic particles. $p_{\max }\left(p_{i n j}\right)$ is the maximum (injected) particle momentum. The growth timescale has to be shorter than the advection timescale towards the shock $\tau_{a d v}=\kappa / V_{s h}^{2}$. The latter is calculated for a diffusion coefficient $\kappa=\eta \kappa_{B}>\kappa_{B}$ taken in the background MF (Eq 2.2) and $\kappa_{B}=c r_{L} / 3$. We find: $\tau_{a d v}=[0.24$ day $] \times \eta \mathrm{E}_{\mathrm{PeV}} \times \mathrm{t}_{\text {day }}^{1.17}$ The condition $\tau_{N R-s t}<\tau_{a d v}$ is necessary but not sufficient for the instability to develop. The magnetic fluctuations produced by the instability uncovered by [15] produce small scale perturbations. The wave number corresponding to the maximum growth rate is [13] $k_{\text {Gmax }} r_{\text {Lmax }} \simeq 4 \times 10^{6}$ for SN 1993J. Acceleration and confinement of energetic particles up to a few $\mathrm{PeV}$ requires magnetic fluctuations to be generated at resonant scales $k r_{L} \simeq 1$, as the NR growth rate scales as $k^{1 / 2}$ the MF at the scale of interest grows over a timescales about 2000 times larger than the one obtained in Eq. 2.3. If the background MF is purely toroidal $\eta<1$, and the instability may not have time to develop.

The $\mathrm{R}$ instability can build up over the NR one [14], with the ratio of the magnetic energies reaching $\sqrt{\xi_{C R} c / V_{s h}} \sim 0.95$ in our case. The growth timescale for the $\mathrm{R}$ instability is however longer than the rate given by Eq. 2.3. We have [13] $\tau_{R-s t} \simeq \sqrt{\pi \sigma / 8} / r_{L m a x}$ with $\sigma \sim 3 \times 10^{16} \mathrm{~cm}^{2} / \mathrm{s}^{2}$ in the conditions that prevail for SN 1993J. This leads to a growth rate $\tau_{R-s t} \simeq 16 \tau_{N R-s t}$ at 1 $\mathrm{PeV}$. The NR instability can be driven to non-linear stages and produce large wave numbers. A typical timescale of non-linear saturation of the MF is about $5 \times \tau_{N R, s t}$ [16]. Recently [17] proposed a ponderomotive instability that builds up on the magnetic fluctuations by the NR streaming instability. We can evaluate the growth time-scale of such long-wavelength modes: $\tau_{L W}=$ $[0.29$ day $] \times \sqrt{\left(\frac{\phi / 15}{\xi_{\mathrm{CR}} / 0.05}\right)} \times \frac{1}{\sqrt{\mathrm{u}_{\mathrm{sh}, 93 \mathrm{~J}}^{3} \mathrm{~A}_{10}}} \times \mathrm{E}_{\mathrm{PeV}} \mathrm{t}_{\text {days }}$. The parameter $A=B_{N R}^{2} / B_{0}^{2}>1$ is the level of magnetic energy produced by the small scale instability with respect to the background CSM and is normalized to 10. Long wavelengths are produced on timescales shorter than $\tau_{a d v}$ for $k r_{L, \max } \simeq 1$.

\subsubsection{Turbulence driven instabilities}

Stellar winds of massive stars are subject to strong fluid instabilities that can lead to turbulent motions [18]. Turbulent density and magnetic fluctuations at SN shocks can result in MF amplification $[19,20]$. In the latter work the presence of CRs is not necessary to produce the magnetic field 
amplification. The MF growth time is controlled by the coherence length of the turbulent spectrum $\mathrm{L}$ and the fluid velocity $u_{s h}$. For SN1993J, $L / u_{s h} \sim[0.3$ year $] \mathrm{L}_{0.01 \mathrm{pc}} / \mathrm{u}_{\mathrm{sh}, 93 \mathrm{~J}}$ hence the magnetic field has to grow over a fraction of $10^{-2}$ of this timescale.

\subsection{Maximum cosmic ray energies}

Immediately after SN outburst, the maximum energy is likely limited by the SNR age. Balancing the age with the acceleration time gives $\tau_{a c c}=g(r) \kappa_{u} / u_{s h}^{2}$, with $r$ the shock compression ratio, and $g(r)=3 r /(r-1) \times\left(\kappa_{d} / \kappa_{u} r+1\right)$. We use $\kappa_{d}=\kappa_{u} / \sqrt{11}$, corresponding to a tangled MF whose tangential component is compressed by a factor 4 [21]. The maximum energy is $E_{\text {max }, a g e, P e V} \simeq \frac{12.3}{\eta g(r)} \times\left(1-t_{\text {day }}^{-0.17}\right)$. But rapidly (see \$2.1) the streaming instability amplifies the $\mathrm{MF}$, and the non-linear process produces a MF at saturation. The typical saturation value [15] is:

$$
B_{\text {sat }}=[16 \text { Gauss }] \times \sqrt{\frac{\xi_{\mathrm{CR}} / 0.05}{\phi / 15}} \times \mathrm{t}_{\text {days }}^{-1} .
$$

This value is within a factor of 2 of the MF derived in the upstream medium from Eq.2.1 using a compression ratio $r=4$. If only the NR instability is at work building the MF, the maximum particle energy is then fixed by a condition over the CR areal charge [16] that produces $\int \tau_{N R-s t}^{-1} d t=6.8$. The latter value corresponds to the amplification of the equipartition MF to the value deduced from radio observations. In that case: $E_{\max , N R, P e V} \sim 1 \times t_{d a y}^{-0.17}$. In the case of long wavelengths fluctuations produced by the ponderomotive instability, the maximum energy is fixed by geometrical losses. The maximum energies are obtained with a diffusion coefficient expressed in the amplified field and compared to $\eta_{e s c} R_{s h} u_{s h}$. In order to derive a time dependence of the maximum energy the time dependence of the amplified field has to be specified, for which we rely on the estimate given in Eq. 2.4. This gives: $E_{\max , L W, P e V} \sim 55\left(\frac{\eta_{\text {esc }}}{0.1}\right) \times t_{\text {day }}^{-0.34}$. Apart from the time dependence of $B_{\text {sat }}$, this value is optimistic since the highest energy particles may feel a MF lower than $B_{\text {sat }}$ [22]. The maximum cosmic ray (proton) energy at any given time is the minimum of all the above limits. In all cases, PeV energies are possible days after the outburst.

\subsection{Cosmic-ray spectral evolution}

We follow the prescription of T09 regarding the time evolution of the CR energy content. The proton particle spectrum is assumed to follow a power-law with a spectral index $s=2$ and an exponential cutoff at $E_{\max }(t)$, the maximum particle energy as discussed in $\S 2.2$. In the case of secondary electrons and positrons produced in the proton-proton interactions, we solve a one-zone energy equation to calculate the time evolution of their energy distribution $N(E, t): \partial_{t} N(E, t)+$ $\partial_{E}(\dot{L}(E) N(E, t))=Q(E, t)$, where $\dot{L}$ includes the synchrotron losses for secondary electrons and positrons in the post-shock region.

\section{Gamma-Ray production}

We derive multi-wavelength time dependent spectra, in the context of MFA, driven by the instabilities discussed in section $\$ 2.1$. We consider only proton-proton interactions, as inverse Compton or bremsstrahlung radiation have been found negligible in the $\mathrm{GeV}-\mathrm{TeV}$ range explored 
here. Inverse Compton process is highly disfavored due to the strong magnetic field at the forward shock. Gamma-rays can be absorbed by different soft photon fields to produce electron-positron pairs. The main photon source is the SN photosphere, described in the case of SN 1993J by [23].

\subsection{Gamma-gamma absorption}

We have performed a full calculation of the gamma-gamma opacity $\tau_{\gamma \gamma}$ including geometrical effects due to the anisotropic interaction (Renaud et al. 2015, in prep). The final gamma-ray flux is the unabsorbed flux $F_{\gamma, \text { un }}$ times an attenuation factor $\exp \left(-\tau_{\gamma \gamma}\left(E_{\gamma}\right)\right)$. Gamma-gamma absorption is strong just after the outburst as the interaction in nearly isotropic, but thereafter drops as the ratio of the forward shock radius to the photosphere radius reaches $\sim 3$, which happens after $\sim 5$ days.

\subsection{Cherenkov Telescope Array detectability}

The time dependent gamma-ray spectra in the very-high energy $(100 \mathrm{GeV}<\mathrm{E}<100 \mathrm{TeV})$ gamma-ray domain are displayed in Figure 1. A source like SN 1993J would be easily detected by CTA above $1 \mathrm{TeV}$ in $20 \mathrm{~h}$ of observing time. The best time window to detect a gamma-ray signal is between a week and a month after the outburst. Prior to a week the source is optically thick to gamma-rays, but the gamma-gamma opacity decreases rapidly due to anisotropic effects. After a month the gamma-ray signal becomes too faint due to the decrease in density of the medium.
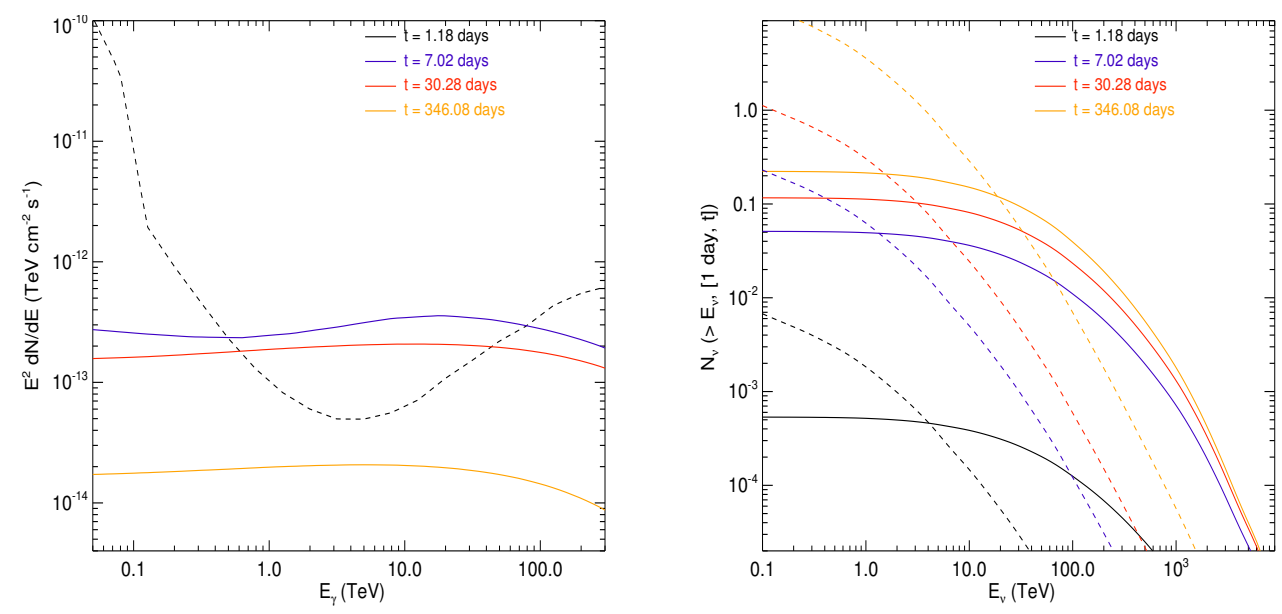

Figure 1: [left] Time dependent spectra at 4 different times after outburst. Dotted lines - expected CTA sensitivity in $50 \mathrm{~h}$. At $\mathrm{t}=1.18$ days, the source is optically thick to gamma rays, and the flux is below the given range. [Right] Time dependent neutrinos flux above an energy E expected by a KM3NeT-like instrument (continuous lines) at 4 different times after outburst. Dotted lines - atmospheric neutrino backgrounds.

\subsection{Neutrino signal}

Neutrinos are by-products of pion production. The expected flux of neutrinos detected by an instrument equivalent to KM3NeT is displayed in Figure 1. We find that at best about 0.1 neutrinos could be expected from a SN 1993J type event above $10 \mathrm{TeV}$ by summing the spectra between 1 and 30.28 days after the outburst, with 0.03 background neutrinos. A signal of one neutrino from such a source requires a source within $\sim 1 \mathrm{Mpc}$, or a gamma-ray signal ten times stronger. 


\section{Discussion \& Conclusions}

SNe produce multi-PeV particles through the combination of: fast shocks $(\mathrm{v} \sim 0.05 \mathrm{c})$, high density CSM produced by stellar winds, and low wind magnetizations. High degree of CSM ionization eases the particle acceleration process. Assuming that the background MF has a turbulent component, different instabilities driven by the acceleration process can grow over intra-day timescales. This model is consistent with the strength of the MF seen in SN 1993J.

Parameters affecting the early gamma-ray emission from $\mathrm{SNe}$ include the ratio of the mass loss rate to the wind velocity $\left(\dot{M} / v_{w}\right)$, which fixes the CSM medium density and affects the CR driven instability growth rate. The shock velocity controls the growth rate of the instabilities and the acceleration timescale. The degree of ionization [24] is important for the particle acceleration efficiency and may also produce element dependent CR spectra if the ionization is partial. The background MF controls partly the local magnetization and the shock obliquity. The SN luminosity controls the gamma-gamma absorption process.

Only about 5-6\% of the local core-collapse SNe have been classified as Type IIb such as SN 1993J [25]. There appear to be two classes of IIb SNe, with compact and extended progenitors [26]. It is likely that only the ones with extended radii and higher mass-loss rates such as 1993J are the more likely candidates for detecting $\gamma$-ray emission, thus further reducing the sample. Type IIn $\mathrm{SNe}$ are probably more promising targets for gamma-ray telescopes in terms of high ambient density without significantly reduced velocities. In the case of the IIn SN 1996cr, it has been deduced that the shock was interacting with a shell of density $\sim 10^{5} \mathrm{~cm}^{-3}$ [27] a few years after explosion. This provides a high density target for producing $\gamma$-rays via pion production (although perhaps not as dense as suggested by some authors [28] for other SNe). Such Type IIn SNe would probably also be promising targets for detecting neutrino emission from secondaries. Unfortunately these sources are even less frequent than the IIb SNe. SN IIP comprise the largest class of core-collapse $\mathrm{SNe}$, making up around half the total. They arise from RSG stars, which have wind mass-loss rates ranging from $10^{-7}$ to $10^{-4} \mathrm{M}_{\odot} \mathrm{yr}^{-1}$ [29]. However, observationally most IIPs appear to arise from the lower end of RSG stars [25] and are less luminous in X-rays [24], suggesting that only the RSG stars with initial masses below $\sim 17-19 \mathrm{M}_{\odot}$, with correspondingly lower mass-loss rates [29], explode to become Type IIP SNe. The rare Ib/Ic SNe harbor the fastest shock waves, but arise from Wolf-Rayet progenitors, which have wind velocities two orders of magnitude greater than RSGs, and therefore should have a correspondingly lower wind density. On the other hand, their $\mathrm{X}$-ray flux is presumed to be due to Inverse Compton or synchrotron emission [30], suggesting accelerated electrons, so it is possible that the shocks are capable of also accelerating protons to high energies. W-R stars are surrounded by low density wind-blown bubbles bordered by a high density shell. If the shell is formed soon before the explosion, as is the case of the SN 2006jc [31], then it provides a good target for accelerated protons to collide with. Such W-R stars may be good candidates for detecting gamma-ray emission in the early phases. There may be SNe similar to SN 1987A, whose progenitor, a blue-supergiant, had a very low mass-loss rate wind [32], but which shows evidence for a dense HII region with density of order 200 particles $\mathrm{cm}^{-3}$ [33], surrounded by a dense circumstellar ring with density $\sim 10^{4}$ particles $\mathrm{cm}^{-3}$ at a distance of $\sim 0.2 \mathrm{pc}$ from the SN. Finally, the class of super-luminous SNe, especially those that are H-rich [34], may be interacting with extremely dense environments. High densities close in to the star could again provide target 
material for proton-proton collisions and detectable $\gamma$-ray emission at an early age.

Acknowledgements This research collaboration is supported by a grant from the FACCTS program to the University of Chicago (PI: VVD; Co-I: MR, Univ of Montpellier). We are grateful to this program for funding travel between Chicago and Montpellier for VVD and MR.

\section{References}

[1] P. Blasi, The origin of galactic cosmic rays, AARs 21 (Nov., 2013) 70, [arXiv: 1311.7346 ].

[2] A. M. Hillas, The Origin of Ultra-High-Energy Cosmic Rays, ARAA 22 (1984) 425-444.

[3] A. M. Bykov, Particle Acceleration and Nonthermal Phenomena in Superbubbles, SSR 99 (Oct., 2001) 317-326.

[4] E. Parizot, A. Marcowith, E. van der Swaluw, A. M. Bykov, and V. Tatischeff, Superbubbles and energetic particles in the Galaxy. I. Collective effects of particle acceleration, AA 424 (Sept., 2004) 747-760, [astro-ph/0405531].

[5] G. Ferrand and A. Marcowith, On the shape of the spectrum of cosmic rays accelerated inside superbubbles, AA $\mathbf{5 1 0}$ (Feb., 2010) A101, [arXiv: 0911.4457 ].

[6] V. Tatischeff, Radio emission and nonlinear diffusive shock acceleration of cosmic rays in the supernova SN 1993J, AA 499 (May, 2009) 191-213, [arXiv: 0903.2944 ].

[7] J. R. Maund and S. J. Smartt, The Disappearance of the Progenitors of Supernovae 1993J and 2003gd, Science 324 (Apr., 2009) 486-, [arXiv: 0903 . 3772].

[8] O. D. Fox, K. Azalee Bostroem, S. D. Van Dyk, A. V. Filippenko, C. Fransson, T. Matheson, S. B. Cenko, P. Chandra, V. Dwarkadas, W. Li, A. H. Parker, and N. Smith, Uncovering the Putative B-star Binary Companion of the SN1993J Progenitor, ApJ 790 (July, 2014) 17, [arXiv: 1405.4863 ].

[9] C. Fransson, P. Lundqvist, and R. A. Chevalier, Circumstellar Interaction in SN 1993J, ApJ 461 (Apr., 1996) 993-+.

[10] P. Chandra, A. Ray, and S. Bhatnagar, The Late-Time Radio Emission from SN 1993J at Meter Wavelengths, ApJ 612 (Sept., 2004) 974-987, [a stro-ph/ 040544 8].

[11] C. Fransson and C. Björnsson, Radio Emission and Particle Acceleration in SN 1993J, ApJ 509 (Dec., 1998) 861-878, [astro-ph/].

[12] A. R. Bell and S. G. Lucek, Cosmic ray acceleration to very high energy through the non-linear amplification by cosmic rays of the seed magnetic field, MNRAS 321 (Mar., 2001) 433-438.

[13] E. Amato and P. Blasi, A kinetic approach to cosmic-ray-induced streaming instability at supernova shocks, MNRAS 392 (Feb., 2009) 1591-1600, [arXiv: 0806.1223 ].

[14] G. Pelletier, M. Lemoine, and A. Marcowith, Turbulence and particle acceleration in collisionless supernovae remnant shocks. I. Anisotropic spectra solutions, AA 453 (July, 2006) 181-191, [astro-ph/0603461].

[15] A. R. Bell, Turbulent amplification of magnetic field and diffusive shock acceleration of cosmic rays, MNRAS 353 (Sept., 2004) 550-558.

[16] A. R. Bell, K. M. Schure, B. Reville, and G. Giacinti, Cosmic-ray acceleration and escape from supernova remnants, MNRAS 431 (May, 2013) 415-429, [arXiv: 1301 . 7264 ]. 
[17] A. M. Bykov, S. M. Osipov, and D. C. Ellison, Cosmic ray current driven turbulence in shocks with efficient particle acceleration: the oblique, long-wavelength mode instability, MNRAS $\mathbf{4 1 0}$ (Jan., 2011) 39-52, [arXiv:1010.0408].

[18] V. V. Dwarkadas, Turbulence in wind-blown bubbles around massive stars, Physica Scripta Volume T 132 (Dec., 2008) 014024, [arXiv: 0810.4361$].$

[19] A. Beresnyak, T. W. Jones, and A. Lazarian, Turbulence-Induced Magnetic Fields and Structure of Cosmic Ray Modified Shocks, ApJ 707 (Dec., 2009) 1541-1549, [arXiv: 0908.2806 ].

[20] J. Giacalone and J. R. Jokipii, Magnetic Field Amplification by Shocks in Turbulent Fluids, ApJL 663 (July, 2007) L41-L44.

[21] A. Marcowith and F. Casse, Postshock turbulence and diffusive shock acceleration in young supernova remnants, AA $\mathbf{5 1 5}$ (June, 2010) A90, [arXiv: 1001.2111 ].

[22] V. Ptuskin, V. Zirakashvili, and E.-S. Seo, Spectrum of Galactic Cosmic Rays Accelerated in Supernova Remnants, ApJ 718 (July, 2010) 31-36, [arXiv: 1006.0034 ].

[23] J. R. Lewis, N. A. Walton, W. P. S. Meikle, R. Martin, R. J. Cumming, R. M. Catchpole, M. Arevalo, R. W. Argyle, C. R. Benn, P. S. Bunclark, and H. O. e. a. Castaneda, Optical Observations of Supernova 1993J from La-Palma - Part One - Days 2 TO 125, MNRAS 266 (Jan., 1994) L27.

[24] V. V. Dwarkadas, On the lack of X-ray bright Type IIP supernovae, MNRAS 440 (May, 2014) 1917-1924, [arXiv:1402.5150].

[25] S. J. Smartt, Progenitors of Core-Collapse Supernovae, ARAA 47 (Sept., 2009) 63-106, [arXiv:0908.0700].

[26] R. A. Chevalier and A. M. Soderberg, Type IIb Supernovae with Compact and Extended Progenitors, ApJL 711 (Mar., 2010) L40-L43, [arXiv:0911.3408].

[27] V. V. Dwarkadas, D. Dewey, and F. Bauer, Bursting SN 1996cr's bubble: hydrodynamic and X-ray modelling of its circumstellar medium, MNRAS 407 (Sept., 2010) 812-829, [arXiv: 1005.1090 ].

[28] K. Murase, T. A. Thompson, B. C. Lacki, and J. F. Beacom, New class of high-energy transients from crashes of supernova ejecta with massive circumstellar material shells, PRD 84 (Aug., 2011) 043003, [arXiv:1012.2834].

[29] N. Mauron and E. Josselin, The mass-loss rates of red supergiants and the de Jager prescription, AA 526 (Feb., 2011) A156, [arXiv:1010.5369].

[30] R. A. Chevalier and C. Fransson, Circumstellar Emission from Type Ib and Ic Supernovae, ApJ 651 (Nov., 2006) 381-391, [astro-ph/].

[31] R. J. Foley, N. Smith, M. Ganeshalingam, W. Li, R. Chornock, and A. V. Filippenko, SN 2006jc: A Wolf-Rayet Star Exploding in a Dense He-rich Circumstellar Medium, ApJL 657 (Mar., 2007) L105-L108.

[32] R. A. Chevalier and V. V. Dwarkadas, The Presupernova H II Region around SN 1987A, ApJL 452 (Oct., 1995) L45-+.

[33] D. Dewey, V. V. Dwarkadas, F. Haberl, R. Sturm, and C. R. Canizares, Evolution and Hydrodynamics of the Very Broad X-Ray Line Emission in SN 1987A, ApJ 752 (June, 2012) 103, [arXiv:1111.5314].

[34] M. Nicholl, S. J. Smartt, A. Jerkstrand, and C. Inserra, On the diversity of super-luminous supernovae: ejected mass as the dominant factor, ArXiv e-prints (Mar., 2015) [arXiv:1503.0331]. 